\title{
Simultaneous strain and temperature multipoint sensor based on microstructured optical fiber
}

\author{
Aitor Lopez-Aldaba, Jean Louis Auguste, Raphael Jamier, Philippe Roy and Manuel Lopez-Amo, \\ Senior Member, IEEE.
}

\begin{abstract}
In this paper, a new sensor system for simultaneous and independent multipoint strain and temperature measurements is presented. The interrogation of the sensing heads has been carried out by monitoring their FFT phase variations. In particular, two of each microstructured optical fiber (MOF) cavity interference frequencies were used for the measures. This method is independent of the signal amplitude and also avoids the necessity of tracking the wavelength evolution in the spectrum, which can be a handicap when there are multiple interference frequency components with different sensitivities. The sensing heads present birefringent and multimodal properties and therefore both characteristics lead to their own interference with different properties and sensitivities. The multiplexing capability of the sensing heads and the interrogator method has also been tested and validated. Sensors were operated within a range of temperature $30^{\circ} \mathrm{C}-80^{\circ} \mathrm{C}$ and a deformation of $\sim 450 \mu \varepsilon$ was applied. Crosstalk between measurements can be corrected through simple math operations leading to independent and crosstalk-free multipoint and multiparameter sensors.
\end{abstract}

Index Terms - microstructured optical fiber, fiber sensor, temperature sensing, strain sensing, simultaneous sensing, multiparameter system, multiplexing.

\section{INTRODUCTION}

ENSORS based on optical fibers have proved to be very useful measuring different parameters such as strain, temperature, curvature, displacement, pressure, refractive index, electric field and gases, among others. In particular, temperature, strain and curvature sensors have grown significantly in importance for health monitoring of aerospace, marine or civil structures, or in fabrication processes [1,2]. As

This paragraph of the first footnote will contain the date on which you submitted your paper for review. Financial support from the Spanish Comisión Interministerial de Ciencia y Tecnología within projects TEC201676021-C2-1-R and TEC2013-47264-C2-2-R. Cost action MP1401 and FEDER funds from the European Union are also acknowledged.

A. Lopez-Aldaba and M. Lopez-Amo are with the Department of Electronic and Electronic Engineering and ISC, Universidad Publica de Navarra, E-31006 Pamplona, Spain (e-mail: aitor.lopez@unavarra.es; mla@unavarra.es)

J.-L. Auguste, R. Jamier and P. Roy are with the Fiber Photonics Department, UMR CNRS/University of Limoges 7252, 123 Avenue Albert Thomas, 87060 Limoges cedex, France (e-mail: jean-louis.auguste@xlim.fr; raphael.jamier@xlim.fr; philippe.roy@xlim.fr). it is well-known, optical fiber sensor presents relevant advantages such as its immunity to electromagnetic interference, small size, reliability, remote sensing ability and multiplexing capability.

Since the first experiments with microstructured optical fibers (MOFs), they have shown improved characteristics over conventional optical fibers and great potential for sensing applications [3]. Different geometries have been proposed for this kind of special fibers. Among them, suspended-core MOFs present relatively large air holes surrounding a small core (typically few $\mu \mathrm{ms}$ of diameter) resembling to be suspended along the fiber length and maintained by small width silica bridges. Different pure silica suspended-core fibers have been applied for instance in temperature [4] curvature [5] and gas sensing [6]. Moreover, there are photonic crystal fibers (PCF) sensors for simultaneous strain and temperature measure based on the detection of different sensitivities of a modal interferometer with a fiber Bragg grating (FBG) [7], with resolutions of $0.27^{\circ} \mathrm{C}$ and $9.1 \mu \varepsilon$, or in combination with a long period grating (LPG) [8], with resolutions of $1.5^{\circ} \mathrm{C}$ and $5.2 \mu \varepsilon$. Further presented sensors were based on the different interference components detection, which had different sensitivities to strain and temperature. In the reference [9] a new design based on clover geometry PCF in a Sagnac configuration was used showing resolutions of $2^{\circ} \mathrm{C}$ and $11 \mu \varepsilon$. Also in the reference [10] a Hi-Bi PCF was used, in a Sagnac configuration too, achieving resolutions of $1.5^{\circ} \mathrm{C}$ and $4.7 \mu \varepsilon$.

Multimode interferometers (MMI) are a particular case of fiber optic sensors and have been extensively considered with standard multimode fibers for sensing of curvature [11], strain, temperature [12] as well as for simultaneous parameter measurements [13]. The appearance of PCF has allowed continuing the investigation of MMI associated now to novel fiber structures with distinct characteristics.

Standard highly birefringent (HB) fibers with elliptical-core or stress-applying elements have been successfully used as active elements of fiber optic sensors for measuring numerous physical parameters such as strain, temperature and pressure [14-17].

In this work a simultaneous strain and temperature sensor based on a microstructured suspended core fiber is characterized. The sensor comprises a suspended core PCF with one of its two end facets cleaved and spliced to a single 
mode fiber (SMF) and is based on the combination of a multimodal and a birefringent interferences with different fringe patterns. Furthermore, a multiplexing technique related to the interrogation method allows multiplexing several sensors within a single optical channel. The monitoring system shows no crosstalk between measurements and maintains the properties of sensitivity and independence of strain and temperature measurements of the single-sensor system.

\section{EXPERIMENTAL SETUP AND OPERATION PRINCIPLE}

The sensing head was fabricated by splicing a commercial SMF to a four-bridge MOF with its end facets cleaved, using a Fitel S175 fusion splicer with a custom developed fusion program designed for this special MOF and operated in manual mode. This splicing special program must deal with a tradeoff between resistant splicing results and maintaining the inner structure of the MOF. The fiber is composed by four big air holes divided by four thin silica bridges, approximately $900 \mathrm{~nm}$ thick, and presents an elongated core of $3.2 \mu \mathrm{m}$ by $1.07 \mu \mathrm{m}$ exhibiting a double $\mathrm{Y}$ shape. This specific shape provides great possibilities for interferometric sensing with multimode and birefringent features. The cross-section of the four-bridge MOF and its core details are presented in Fig. 1.
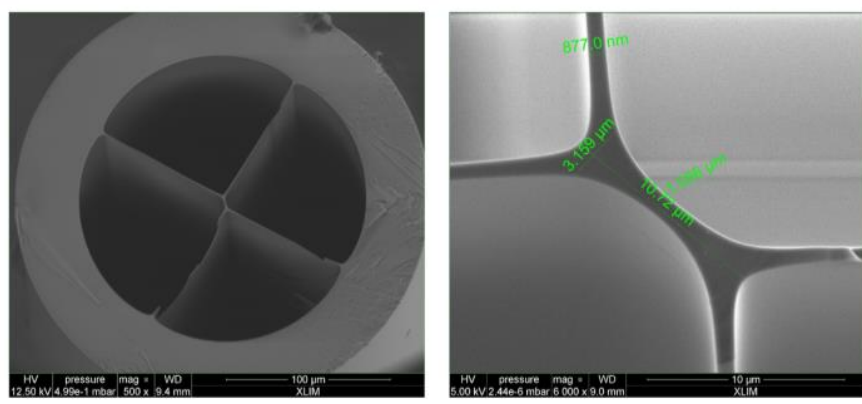

Fig. 1. Four-bridge MOF cross section and close-up on the core details.

Fig. 2 a) shows the two different experimental set-ups used to characterize the single four-bridge MOF Fabry-Perot interferometer. In the first set-up (top of the Fig. 2 a), the sensing head was inserted into an oven where temperature changes (going from $30^{\circ} \mathrm{C}$ to $75^{\circ} \mathrm{C}$ by steps of $5^{\circ} \mathrm{C}$ ) were applied. Fig. 2. a) (bottom) shows the strain testing set-up. In this set-up, point 1 of the fiber was fixed, and a maximum longitudinal strain of $380 \mu \varepsilon$ was applied to point 2 , having the length of the MOF fiber equal to $14.5 \mathrm{~cm}$.

Fig. 2 b) is the equivalent scheme for the multipoint sensing system. In this case two MOF-FP sensing heads were tested simultaneously to verify the independence of measurements. The main difference in this setup compared to the previously described is the insertion of an EDFA to reach optical power stable values and an optical coupler as the multiplexing device. Due to the high transmission losses of the MOF, the inefficient light coupling at the SMF-MOF fiber connection (mainly produced by the core dimensions differences) and the losses related to the coupling ratio of the optical coupler, an optical amplifier is necessary to ensure the optical power stability of both sensors. In this particular case the optical amplifier used was an erbium-doped fiber amplifier (EDFA,
MPB P21) with an optical gain of $17 \mathrm{~dB}$ and a wavelength operation range of $1525-1565 \mathrm{~nm}$.

A 2x2 optical coupler was employed as a multiplexing device. Two different optical couplers with different coupling ratio were tested. Initially a 50:50 optical coupler was chosen to split the optical power equally in both sensing heads (MOFx and MOFy) but it was found out that due to the different length of the MOFs, the shorter one had higher optical power levels (one order of magnitude) than the larger one. To solve this difference and in order to equalize both contributions a 70:30 optical coupler was used with the $70 \%$ arm dedicated to the larger MOF and the $30 \%$ arm to the shorter one.

Two optical 3-port circulators were placed in order to recirculate the signals and allowing the use of a single optical channel of the optical interrogator.
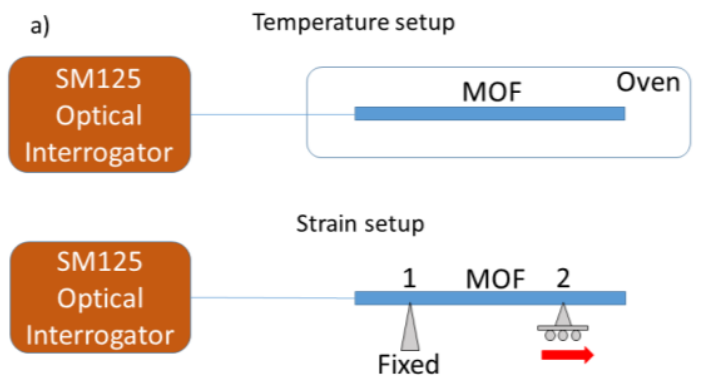

b)
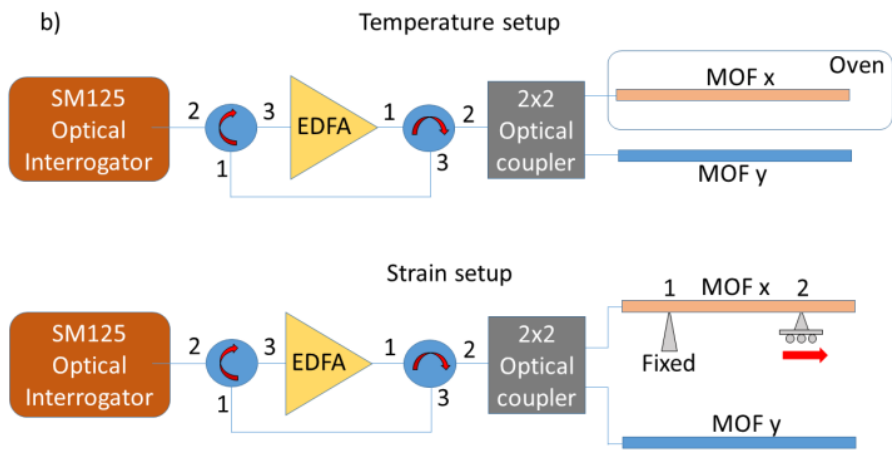

Fig. 2. Schematic representations of the utilized experimental setups.

A commercial interrogator of optical fiber sensors (Smartec SM125) was used to illuminate the network and also to analyze the spectra signals guided through the MOF sensor. It should be noticed that this equipment was originally commercialized for FBG sensors' monitoring and allows to interrogate sensors with a scan frequency of $1 \mathrm{~Hz}$ and a $5 \mathrm{pm}$ resolution. The FFT is computed in MATLAB also every second, providing real-time information of the sensor system [18].

The interrogation technique is based on the Fast Fourier Transform of the sensor's optical spectrum. By monitoring the Fast Fourier Transform phase, wavelength shifts in the optical spectrum can be monitored without the noise influence or signal amplitude variations and this enables multiplexing several sensors within a single interrogator's channel. Optical 
spectrum signal amplitude undesired variations lead to changes in the FFT module amplitude but do not affect the FFT phase.

Three different sensing heads were developed. Sensor0 (S0) was made of $24 \mathrm{~cm}$ of $\mathrm{MOF}$ showing an interferometric spectrum as seen in Fig. 3 a). This optical spectrum is composed by several interferometric contributions. As Fig. 3 b) illustrates, there are three main components. The lowest component located at $\sim 0.27 \mathrm{~nm}^{-1}$ is a Fabry-Perot undesired contribution produced at splice. S0 F1 (related to Sensor 0 Frequency 1) is a result of the birefringence of the MOF due to the asymmetric core of the MOF. The period of this interference is given by:

$$
\Delta \lambda=\left(\mathrm{L}_{\mathrm{B}} \times \lambda\right) / \mathrm{L}
$$

where $\Delta \lambda$ is the interference period spacing, $\mathrm{L}_{\mathrm{B}}=3.96 \times 10^{-4} \mathrm{~m}$ is the MOF simulated beat length [4] and L (expressed in $\mathrm{m}$ ) is the MOF length. In this particular case, sensors are measured in reflection and therefore the fiber length must be multiplied by a factor 2 . As a result, it appears an interference due to the fiber birefringence with frequency located at $0.8 \mathrm{~nm}^{-1}$.
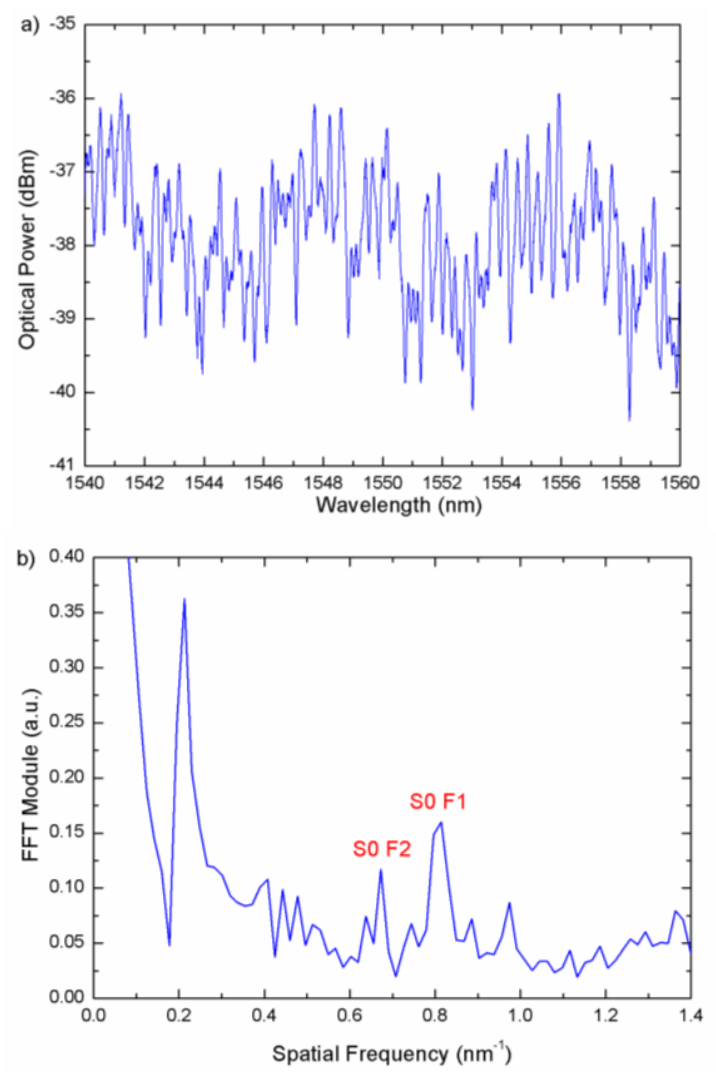

Fig. 3. a) Optical spectrum of S0 and b) its FFT.

S0 F2 is an interference produced as a result of the beating of two modes that propagate in the sensing head fiber which shows a multimode behavior. Other less strong multimodal beating interferences appear which come from the interference of high order modes propagating along the fiber, but these modes are less powerful.
In parallel other two sensing heads were created to multiplex them in a single optical channel showing similar features (two main interferences, the lower one (F1) due to the birefringence and the higher one (F2) due to the multimodal interference) $\mathrm{S} 1$ and $\mathrm{S} 2$, made of $34 \mathrm{~cm}$ and $28 \mathrm{~cm}$ of $\mathrm{MOF}$ fiber respectively.

The operation principle is based on the properties of birefringence and multimodal interferences. Birefringence interference is produced by the beat of the light propagating in $\mathrm{x}$ and $\mathrm{y}$ axis. Therefore, temperature variations affects similarly to both axis and the interference remains almost unaltered. Strain affects in a different manner to both axis depending on the position of the fiber on the fixed points. Multimodal interference is a result of the beat of two propagating modes. Each mode is affected by the refractive index of core and cladding and temperature varies these properties while strain does not (at least not in the same order of magnitude).

\section{EXPERIMENTAL RESULTS}

\section{A. Simultaneous strain and temperature measurements}

In order to verify the operation of the sensor S0, the strain and temperature performances of the MOF sensing head were experimentally carried out.

On the one hand, the temperature characterization was carried out by using an oven in the temperature range going from $30^{\circ} \mathrm{C}$ to $75^{\circ} \mathrm{C}$ with $5^{\circ} \mathrm{C}$ per step applying $0 \mathrm{~nm}$ of strain to it. On the other hand, strain characterization was performed using two translation stages at $14.5 \mathrm{~cm}$ from each other, one remaining fixed (point 1 in Fig. 2 a) while the other moves applying strain (steps of $17 \mathrm{~nm}$ ) to the fiber (point 2 in Fig. 2 a) at room temperature $\left(23^{\circ} \mathrm{C}\right)$. Both spatial frequencies S0 F1 and S0 F2 were simultaneously monitored to ensure the measurements independence.

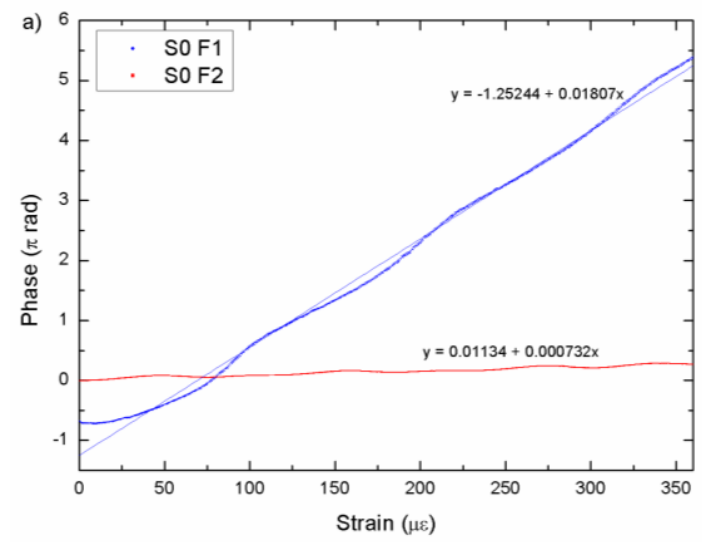




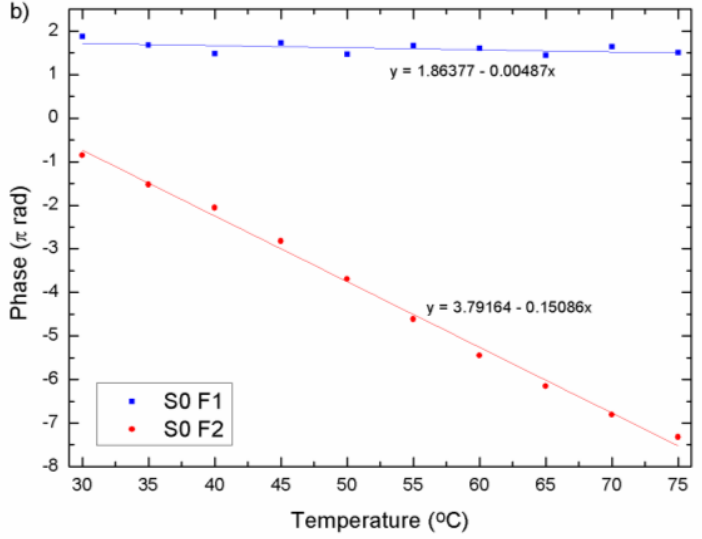

Fig. 4. Performance of the spatial frequencies towards strain and temperature variations: a) strain applied and b) temperature variations applied.

Fig. 4 illustrates the FFT phase response of both frequencies towards strain and temperature variations. As it can be noticed the S0 F1 phase presents linear response to strain variations (due to the lack of tightness in the fiber during the strain measurements in the range located from 0 to $50 \mu \varepsilon$, the measured results show a slight deviation from the linear fit) and low sensitivity to temperature variations. This is due to the birefringent character of the interference related to the F1 spatial frequency, showing sensitivities to strain and temperature of $0.018 \pi \mathrm{rad} / \mu \varepsilon$ and $-0.0048 \pi \mathrm{rad} /{ }^{\circ} \mathrm{C}$ respectively and a resolution of $0.262 \mu \varepsilon$. Overhanging these results to the optical domain, sensitivities to strain and temperature are $-19.1 \mathrm{pm} / \mu \varepsilon$ and $0.615 \mathrm{pm} /{ }^{\circ} \mathrm{C}$ respectively.

Component S0 F2 shows complementary results. On the contrary to S0 F1, it presents linear response to temperature variations and very low sensitivity to strain variations, because of the multimodal behavior of the MOF fiber. Sensitivities to strain and temperature are respectively of $0.00073 \pi \mathrm{rad} / \mu \varepsilon$ and $-0.15 \pi \mathrm{rad} /{ }^{\circ} \mathrm{C}$ and resolution of $0.039^{\circ} \mathrm{C}$. Overhanging the results to the optical domain sensitivities to strain and temperature are $-0.74 \mathrm{pm} / \mu \varepsilon$ and $127.5 \mathrm{pm} /{ }^{\circ} \mathrm{C}$ respectively.

With these results the contribution of both interferences can be written:

$$
\begin{aligned}
& \Delta \lambda_{\text {SO F1 }}=-0.0048 \pi \Delta \mathrm{T}+0.018 \pi \Delta \varepsilon \\
& \Delta \lambda_{\text {SO F2 }}=-0.15 \pi \Delta \mathrm{T}+0.00073 \pi \Delta \varepsilon
\end{aligned}
$$

These equations reveal it is possible to discriminate the strain and temperature effects by rearranging them in the following way:

$$
\left[\begin{array}{l}
\Delta T \\
\Delta \varepsilon
\end{array}\right]=\frac{1}{0.002711 \pi^{2}}\left[\begin{array}{cc}
0.00073 \pi & -0.018 \pi \\
0.15 \pi & -0.0048 \pi
\end{array}\right]\left[\begin{array}{c}
\Delta \lambda_{S 0} F 1 \\
\Delta \lambda_{S 0} F 2
\end{array}\right]
$$

The temperature and strain measurements based on this approach show resolutions, $\delta \mathrm{T}$ and $\delta \varepsilon$, which were calculated using the method presented in [19] considering that the optical interrogator has a phase resolution of $\delta_{\text {int }}=0.008 \pi$. It turned out values of $\delta \mathrm{T}=0.055^{\circ} \mathrm{C}$ and $\delta \varepsilon=0.045 \mu \varepsilon$, which compare favorably with values previously reported by other authors $[19,20]$.

\section{B. Multipoint simultaneous strain and temperature measurements}

In order to test the capability of these type of sensors to be multiplexed in a single optical interrogator's channel and ensure the independence of the measurements without crosstalk, Sensor1 (S1) and Sensor2 (S2) were developed using the same type of MOF as Sensor 0.

In this case $S 1$ was made using $34 \mathrm{~cm}$ of MOF fiber and S2 used $28 \mathrm{~cm}$ of the same type of fiber, showing interferences due to its birefringent behavior located in the FFT domain at $1.11 \mathrm{~nm}^{-1}$ and $0.93 \mathrm{~nm}^{-1}$ respectively, as theoretically expected from (1).

As it was previously commented, S1 presents higher losses due to the fiber length than S2. Therefore a 70:30 optical coupler was employed to multiplex both sensors with the $70 \%$ arm dedicated for S1 and the 30\% arm for S2 resulting the optical spectrum and its FFT shown in Fig. 5. Furthermore, an EDFA with $17 \mathrm{~dB}$ of gain was necessary to achieve a stable power level. Without the EDFA, the multiplexed FFT components were almost at noise level and instabilities made measurements impossible.
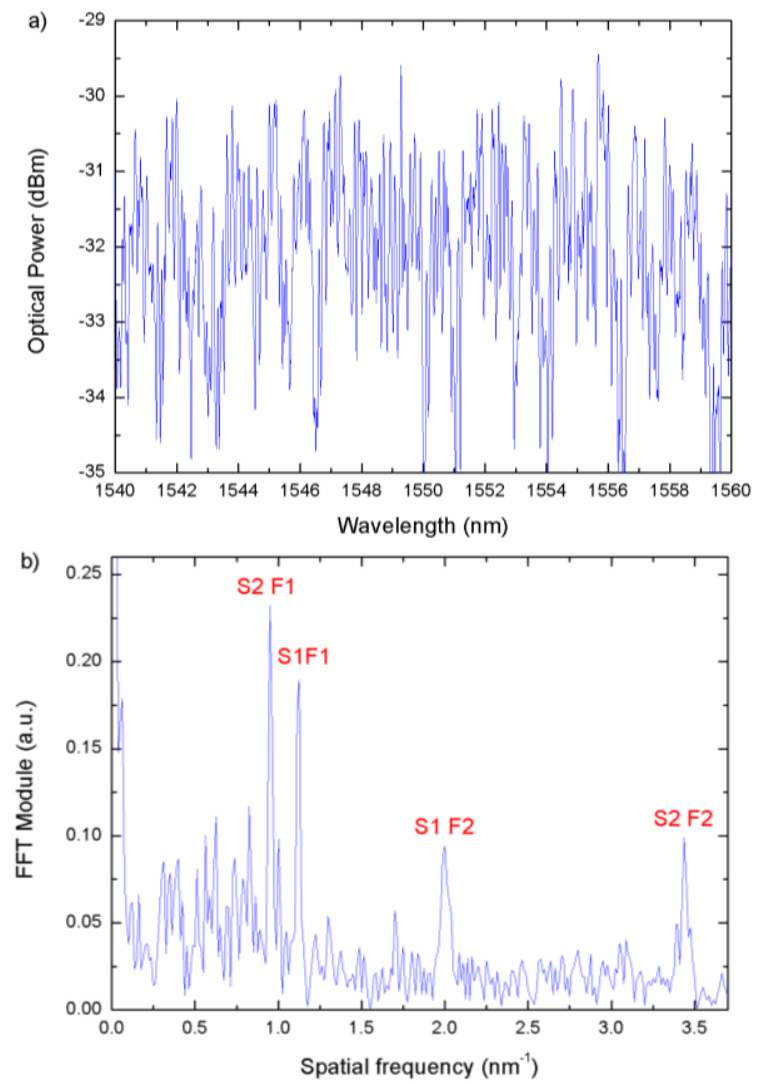

Fig. 5. a) Optical spectrum of multiplexed S1 and S2 and b) its FFT.

The setup for strain and temperature tests was the one described in Fig. 2 b). For temperature variations Sensor1 was placed inside an oven and temperature variations between 30 $80^{\circ} \mathrm{C}$ were applied, while Sensor 2 remained at constant room temperature $\left(22^{\circ} \mathrm{C}\right)$. Same process was performed with Sensor2 inside the oven and Sensor1 at constant room temperature. All the spatial frequencies S1 F1, S1 F2, S2 F1 and S2 F2 were simultaneously monitored. 
For strain tests the method followed was the same as used in the characterization of S0. Around $450 \mu \varepsilon$ of longitudinal strain was applied to one sensor while the other rested on the optical bench also at room temperature; and vice versa.

Fig. 6 shows the results for both sensors being monitored simultaneously. As expected both sensors present similar results as Sensor0 with, on the one hand, the interference related to the birefringence behavior showing high sensitivity to applied strain and low sensitivity to temperature variations and on the other hand, the interference related to the multimode behavior showed sensitivity to temperature and low sensitivity to strain. In addition to these results, the independence of both sensors is proved. Due to the isolation of the spatial frequencies given by the FFT analysis technique each sensor can be measured independently from the other one. In this particular study two sensors where simultaneously tested but a higher number can be multiplexed by choosing correctly the MOF lengths of each one and ensuring a nonoverlapping FFT spectrum.

For Sensor1, S1 F1 presents linear response to strain variations with a sensitivity of $0.0183 \pi \mathrm{rad} / \mu \varepsilon$ and low sensitivity to temperature of $-0.0047 \pi \mathrm{rad} /{ }^{\circ} \mathrm{C}$.
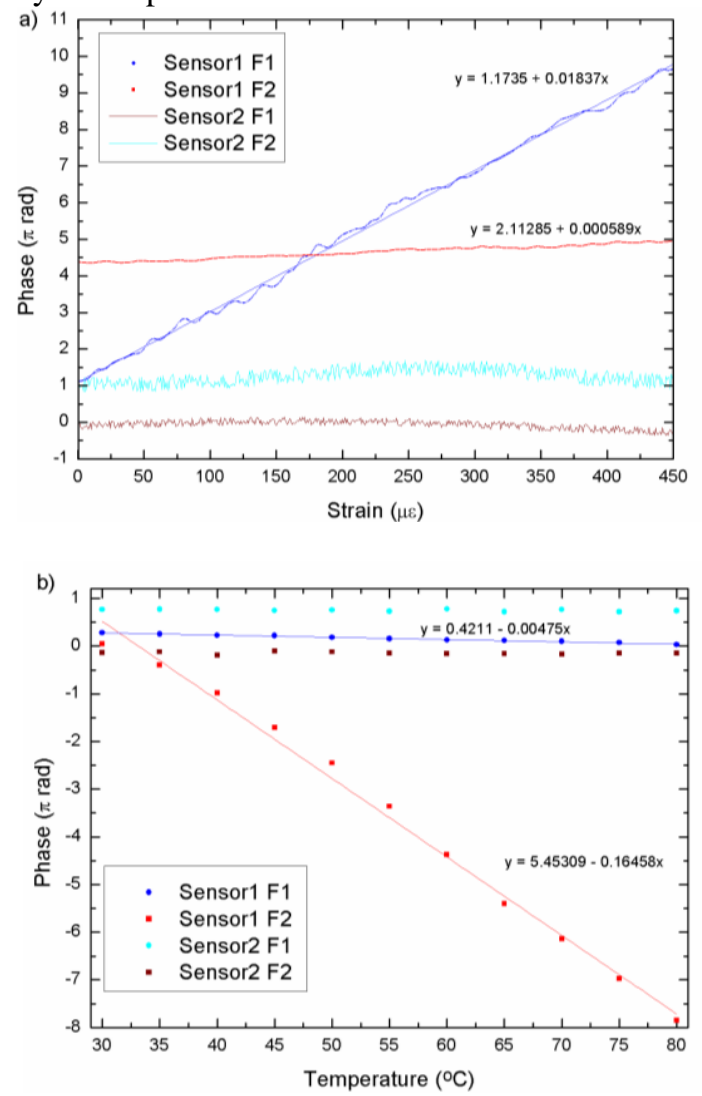
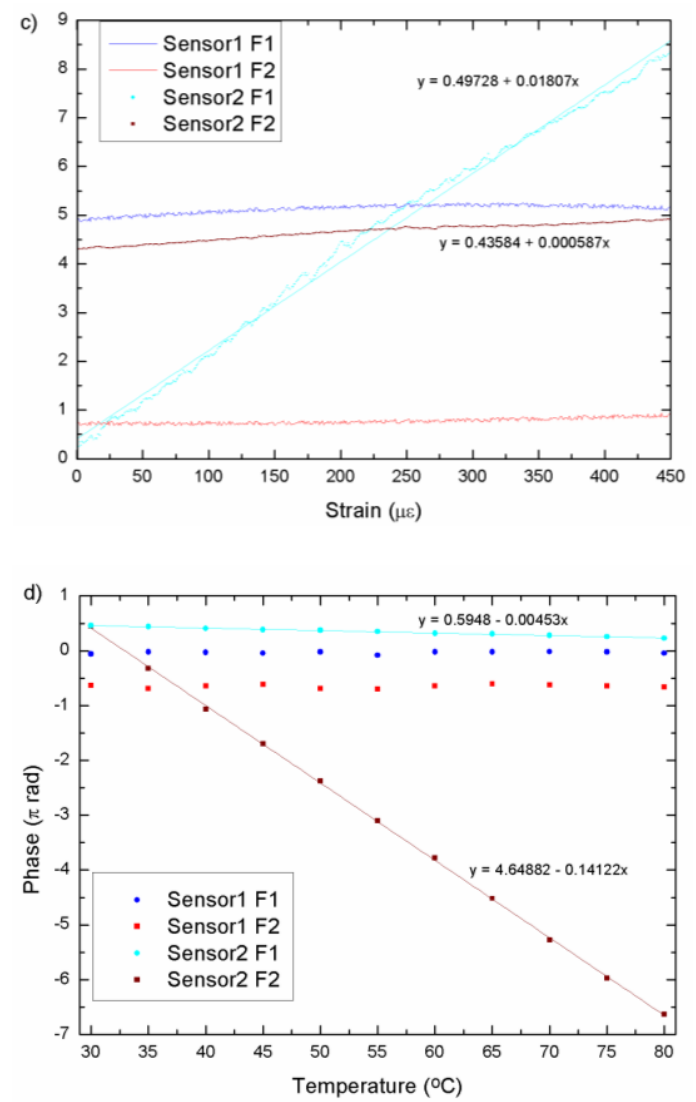

Fig. 6. Performance of the spatial frequencies towards strain and temperature variations: a) strain applied on $\mathrm{S} 1, \mathrm{~b})$ temperature variations applied on S1, c) strain applied on S2 and d) temperature variations applied on S2.

Complementarily S1 F2 presents linear response to temperature variations with sensitivity of $-0.165 \pi \mathrm{rad} /{ }^{\circ} \mathrm{C}$ and $0.00059 \pi \mathrm{rad} / \mu \varepsilon$ for strain measurements. Resolutions for strain in S1 F1 and for temperature in S1 F2 are $0.251 \mu \varepsilon$ and $0.034^{\circ} \mathrm{C}$ respectively.

The contribution of both interferences can be generally written as:

$$
\begin{aligned}
& \Delta \lambda_{\mathrm{S} 1 \mathrm{~F} 1}=-0.0047 \pi \Delta \mathrm{T}+0.0183 \pi \Delta \varepsilon \\
& \Delta \lambda_{\mathrm{S} 1 \mathrm{~F} 2}=-0.165 \pi \Delta \mathrm{T}+0.00059 \pi \Delta \varepsilon
\end{aligned}
$$

Equivalently to the process followed for Sensor0 it is possible to discriminate the strain and temperature influence:

$$
\left[\begin{array}{l}
\Delta T \\
\Delta \varepsilon
\end{array}\right]=\frac{1}{0.003008 \pi^{2}}\left[\begin{array}{cc}
0.00059 \pi & -0.0183 \pi \\
0.165 \pi & -0.0047 \pi
\end{array}\right]\left[\begin{array}{c}
\Delta \lambda_{S 2} F 1 \\
\Delta \lambda_{S 2} F 2
\end{array}\right]
$$

The temperature and strain measurements based on this approach show resolutions of $\delta \mathrm{T}=0.049^{\circ} \mathrm{C}$ and $\delta \varepsilon=0.44 \mu \varepsilon$.

Sensor2 presents similar behavior: S2 F1 shows linear response to strain with sensitivity of $0.018 \pi \mathrm{rad} / \mu \varepsilon$ and $-0.0045 \pi \mathrm{rad} /{ }^{\circ} \mathrm{C}$ to temperature variations. S2 F2 shows linear response to temperature with sensitivity of $-0.14 \pi \mathrm{rad} /{ }^{\circ} \mathrm{C}$ and $0.00059 \pi \mathrm{rad} / \mu \varepsilon$ to strain. The results can be expressed as:

$$
\begin{aligned}
& \Delta \lambda_{\mathrm{S} 2 \mathrm{~F} 1}=-0.0045 \pi \Delta \mathrm{T}+0.018 \pi \Delta \varepsilon \\
& \Delta \lambda_{\mathrm{S} 2 \mathrm{~F} 2}=-0.14 \pi \Delta \mathrm{T}+0.00059 \pi \Delta \varepsilon
\end{aligned}
$$


As previously they can be written in matrix form to isolate each contribution:

$$
\left[\begin{array}{l}
\Delta T \\
\Delta \varepsilon
\end{array}\right]=\frac{1}{0.00251 \pi^{2}}\left[\begin{array}{cc}
0.00059 \pi & -0.018 \pi \\
0.14 \pi & -0.0045 \pi
\end{array}\right]\left[\begin{array}{l}
\Delta \lambda_{S 2 ~ F 1} \\
\Delta \lambda_{S 2 ~ F 2}
\end{array}\right]
$$

This method gives resolutions of $\delta \mathrm{T}=0.071^{\circ} \mathrm{C}$ and $\delta \varepsilon=0.45 \mu \varepsilon$.

\section{CONCLUSIONS}

To summarize, a new sensor system for simultaneous and quasi-independent strain and temperature measurements has been proposed and experimentally demonstrated. The sensing head is based on a microstructured optical fiber used to create a cavity in reflection configuration. The interrogation of the sensing head has been carried out by monitoring the FFT phase variations of two of the MOF cavity interference frequencies. This method is independent from the signal amplitude and also avoids the need to track the wavelength evolution of the spectrum, which can be a handicap when there are multiple interference frequency components with different sensitivities. Furthermore this technique allows to multiplex several sensors within a single optical interrogator's channel with no crosstalk between sensors reducing proportionally the relative cost of the system. Sensors have linear response in temperatures ranged $30-80^{\circ} \mathrm{C}$ and up to $450 \mu \varepsilon$. In the worst case, resolutions of $0.098^{\circ} \mathrm{C}$ and $0.89 \mu \varepsilon$ have been achieved after isolating each contribution.

\section{REFERENCES}

[1] J. M. Lopez-Higuera, L. Rodriguez Cobo, A. Quintela Incera, and A. Cobo, "Fiber optic sensors in structural health monitoring," J. Lightw.Technol., vol. 29, no. 4, pp. 587-608, Feb. 2011.

[2] H. N. Li, D. S. Li, and G. B. Song, "Recent applications of fiber opticsensors to health monitoring in civil engineering," Eng. Struct., vol. 26, pp. 1647-1657, 2004.

[3] A. M. R. Pinto, and M. Lopez-Amo, "Photonic Crystal Fibers for Sensing Applications," Journal of Sensors 2012, 21 (2012).

[4] A. Lopez-Aldaba, A. M. R. Pinto, M. Lopez-Amo, O. Frazão, J. L. Santos, J. M. Baptista, H. Baierl, J. L. Auguste, R. Jamier and P. Roy, "Experimental and Numerical Characterization of a Hybrid FabryPérot Cavity for Temperature Sensing," Sensors 15, 8042-8053 (2015).

[5] O. Frazão, S. F. O. Silva, J. Viegas, J. M. Baptista, J. L. Santos, J. Kobelke, and K. Schuster, "All fiber Mach-Zehnder interferometer based on suspended twin-core fiber," IEEE Photonics Technology Letters, 17(22), 1300-1302 (2010).

[6] A. S. Webb, F. Poletti, D. J. Richardson, and J. K. Sahu, "Suspended-core holey fiber for evanescent- field sensing," Optical Engineering, 46(1), 010503010503 (2007).

[7] B. Dong, J. Hao, C. Y. Liaw, B. Lin, and S. C. Tjin, "Simultaneous strain and temperature measurement using a compact photonic crystal fiber inter-modal interferometer and a fiber Bragg grating," Applied optics, 49(32), 6232-6235 (2010).

[8] T. Li, X. Dong, C. C. Chan, L. Hu, and W. Qian, "Simultaneous strain and temperature measurement based on a photonic crystal fiber modal-interference interacting with a long period fiber grating," Optics Communications, 285(24), 4874-4877 (2012).

[9] R. A. Perez-Herrera, R. M. André, S. F. Silva, M. Becker, K. Schuster, J. Kobelke, M. Lopez-Amo, J. L. Santos, and O. Frazão, "Simultaneous measurement of strain and temperature based on clover microstructured fiber loop mirror," Proc. of SPIE 8421, 84216W-1 (2012).

[10] R. M. Andre, M. B. Marques, P. Roy, and O. Frazao, "Fiber loop mirror using a small core microstructured fiber for strain and temperature discrimination," IEEE Photonics Technology Letters, 22(15), 1120-1122 (2010).

[11]Y. Gong, T. Zhao, Y. Rao, and Y. Wu, "All-fiber curvature sensor based on multimode interference," IEEE Photon. Technol. Lett., vol. 23, no. 11, pp. 679-681, Jun. 2011.

[12] S. M. Tripathi, A. Kumar, R. K. Varshney, Y. B. P. Jumar, E. Marin, and J. Meunier, "Strain and temperature sensing characteristics of singlemodemultimode-single-mode structures," J. Lightw. Technol., vol. 27, no. 13, pp. 2348-2356, Jul. 2009.

[13] Q. Wu, A. M. Hatta, P. Wang, Y. Semenova, and G. Farrell, "Use of a bent single SMS fiber structure for simultaneous measurement of displacement and temperature sensing," IEEE Photon. Technol. Lett., vol. 23, no. 2, pp. 130-132, Jan. 2011.

[14] O. Frazão, J. M. T. Baptista, and J. L. Santos, "Recent advances in high-birefringence fiber loop mirror sensors", Sensors 7 (2007) 2970-2983.

[15] Y. J. Rao, and D. A. Jackson, "Recent progress in fiber optic low-coherence interferometry", Meas. Sci. Technol. 7 (1996) 981-999.

[16]W. J. Bock and W. Urbanczyk, "Temperaturedesensitization of fibre-optic pressure sensor by simultaneous measurement of pressure and temperature", Appl. Opt. 37 (1998) 3897-3901.

[17]W. Urbanczyk and W. J. Bock, "Influence of dispersion on sensitivity of highly birefringent fibers to temperature and hydrostatic pressure", Appl. Opt. 37 (1998) 3176-3180.

[18]D. Leandro, M. Bravo, A. Ortigosa and M. LopezAmo, "Real-time FFT analysis for interferometric sensors multiplexing," J Lightwave Technol. 33(2), 354-360, (2015).

[19] W. Jin, W. C. Michie, G. Thursby, M. Konstantaki, and B. Culshaw, "Simultaneous measurement of strain and temperature: Error analysis," Opt. Eng., vol. 36, pp. 598-609, 1997. 
[20] S. Rota-Rodrigo, M. López-Amo, J. Kobelke, K. Schuster, J. L. Santos and O. Frazão, "Multimodal interferometer based on a suspended core fiber for simultaneous measurement of physical parameters. Journal of Lightwave Technology", 33(12), 24682473 (2015).

\begin{abstract}
Aitor Lopez-Aldaba was born in Navarra, Spain, in July 1988. He received the, Telecommunication Engineering degree from the Universidad Pública de Navarra, Navarra, in 2014. In 2013, he joined the Optical Communications Group, Department of Electrical and Electronic Engineering, Universidad Pública de Navarra. His research interests include fiber optic lasers, optical amplifiers, optical fiber sensor networks, photonic crystal fibers and chemical fiber optic sensors.
\end{abstract}

Manuel López-Amo (M'91-SM'98) was born in Madrid, Spain, in 1960. He received the Telecommunications Engineering and Ph.D. degrees from the Universidad Politécnica de Madrid, Madrid, in 1985 and 1989, respectively. From 1985 to 1996, he belonged to the Photonic Technology Department, Universidad Politécnica de Madrid, where, in 1990, he became an Associate Professor. In 1996, he moved to the Electrical and Electronic Engineering Department, Public University of Navarra, Pamplona, Spain, where he became a Full Professor and is currently the Head of the Optical Communications Group. He has been the Chairman of the Optoelectronic Committee of Spain. He has been the leader of more than 50 research projects and he has coauthored more than 300 works in international refereed journals and conferences related with fiber-optic networks, optical amplifiers, fiber-optic sensors, and integrated optics. $\mathrm{He}$ is a member of the technical committees of the International Conference on Fiber Optic Sensors and the European Workshop on Optical Fiber Sensors, among others. $\mathrm{He}$ is a senior member of the IEEE and member of the Optical Society of America.

Jean-Louis Auguste received a $\mathrm{PhD}$ degree in Optical and High Frequency of Telecommunications from the University of Limoges (France) in 2001. During his thesis, the main areas of activity have been involved theory, design and experimental investigation on optical fibres and more particularly on a design and fabrication of high negative chromatic dispersion fibre. Since December 2000, he is a CNRS Engineer at Xlim research institute where he was, until 2012, in charge (Process
Manager) of the research and development around fabrication of optical fibres - guide structure (PCF) and materials for optical fibres with recent developments around glass synthesis thanks to a European project (VORTEX) that he manages. He works in strong collaboration with researchers of the Fibre Group and is associated to ANR and other European Projects like researcher from Ceramic Laboratory to develop new topics mixing optical glass development and optical fibre applications. By this way in collaboration with researcher from SPCTS (Ceramic Laboratory) he manages a new research project at Xlim around 'original glasses for optical fibre applications'. He has authored or co-authored 45 publications in international journals with referees and 43 papers in international conferences with referees including several invited papers and international seminars plus 4 patent applications. In 2010, he has obtained the degree of HDR, highest diploma of University and giving him the possibility to be Research Director for PhD students. A part of his activity is dedicated to teaching and training students from University and school engineering.

Raphael Jamier was born in Périgueux, France, in 1981. He received the $\mathrm{Ph} . \mathrm{D}$. degree in 2007 from the University of Limoges, France. Since 2008, he has been an Assistant professor at XLIM research institute / University of Limoges, where he has been engaged in design, fabrication and characterisation of specialty optical fibers. His current research activities include the design, fabrication and characterization of photonic crystal fibers for high-power generation at unconventional spectral domains (in particular in the mid-infrared wavelengths). He is also the Deputy Director of the Physics Department of the University of Limoges since 2012. - He has 27 articles as co-author/author in refereed journals, 58 papers in internationally recognized conferences with peer-review system and 2 patents.

Philippe Roy was born in May 1971 in Bellac (France) and received his $\mathrm{Ph}$ Degree in Microwave Electronics and Optoelectronics (speciality Photonics and Electronics Systems) in 1997 in the University of Limoges. He is now a CNRS senior researcher and head of Fibre Photonics group at XLIM, which is a mixed laboratory of University of LIMOGES and CNRS. Since 1998, he is involved in design, fabrication and characterisation of Photonic Crystal fibres (PCFs). More recently, he developed specialty and composite fibres mainly dedicated to advanced fibre sensor systems and to very high power fibre lasers. He develops rare earth doped fibre with complex structure based on an aperiodic design to reach higher power levels without modal instabilities and/or non-conventional emitted spectrum, from visible to $\mathrm{THz}$ domain. 